\title{
Smoking habits in the randomised Danish Lung Cancer Screening Trial with low-dose CT: final results after a 5-year screening programme
}

\author{
Haseem Ashraf, ${ }^{1,2}$ Zaigham Saghir, ${ }^{1}$ Asger Dirksen, ${ }^{1}$ Jesper Holst Pedersen, ${ }^{3}$ \\ Laura Hohwü Thomsen, ${ }^{1}$ Martin Døssing, ${ }^{4}$ Philip Tønnesen ${ }^{1}$
}

\begin{abstract}
${ }^{1}$ Department of Respiratory Medicine, Gentofte University Hospital, Hellerup, Denmark ${ }^{2}$ Department of Radiology, Akershus University Hospital, Lørenskog, Norway ${ }^{3}$ Department of Thoracic Surgery RT, Rigshospitalet, Faculty of Health Sciences, University of Copenhagen, Copenhagen, Denmark ${ }^{4}$ Department of Internal Medicine, Frederikssund Hospital, Frederikssund, Denmark
\end{abstract}

Correspondence to Dr Haseem Ashraf, Department of Respiratory Medicine, Gentofte University Hospital, 2900 Hellerup, Denmark; haseem.ashraf@gmail.com

Received 7 May 2013 Revised 15 October 2013 Accepted 26 December 2013 Published Online First 17 January 2014

\begin{abstract}
Background We present the final results of the effect of lung cancer screening with low-dose CT on the smoking habits of participants in a 5-year screening trial. Methods The Danish Lung Cancer Screening Trial (DLCST) was a 5-year screening trial that enrolled 4104 subjects; 2052 were randomised to annual low-dose CT (CT group) and 2052 received no intervention (control group). Participants were current and ex-smokers ( $\geq 4$ weeks abstinence from smoking) with a tobacco consumption of $\geq 20$ pack years. Smoking habits were determined annually. Missing values for smoking status at the final screening round were handled using two different models.
\end{abstract}

Results There were no statistically significant differences in annual smoking status between the CT group and control group. Overall the ex-smoker rates (CT + control group) significantly increased from $24 \%$ (baseline) to $37 \%$ at year 5 of screening $(p<0.001)$. The annual point prevalence quit rate increased from $11 \%$ to $24 \%$ during the five screening rounds; the ex-smokers' relapse rate remained stable, around $11 \%$, across the same period. Conclusions Screening with low-dose CT had no extra effect on smoking status compared with the control group, but overall the screening programme probably promoted smoking cessation.

Clinical Trial Registration: The DLCST is registered in Clinical Trials.gov Protocol Registration System (identification no. NCT00496977).

\section{INTRODUCTION}

Due to the poor prognosis of lung cancer, the concept of early detection using screening with CT of the thorax has been evaluated in studies in Europe $^{1-3}$ and the USA. ${ }^{4}$ The intention of screening is to detect lung cancer at an earlier stage than usual, and thus reduce mortality from lung cancer in asymptomatic current and ex-smokers. The US National Lung Cancer Screening Trial, which enrolled 50000 participants, reported a significant $20 \%$ reduction in lung cancer mortality, while the all-cause mortality reduction was $6.7 \% .^{6}$

One possible drawback of screening is that it may reduce smokers' motivation to quit by inducing a false sense of safety. ${ }^{7}$ Participants in screening programmes may feel that they are protected against the harmful effects of smoking and may therefore continue to smoke or-even worse-increase the amount they smoke. ${ }^{8}$ Any potential mortality reduction from lung cancer screening would

\section{Key messages}

What is the key question?

- Does screening for lung cancer with low-dose CT of thorax affect smoking behaviour?

What is the bottom line?

- No significant difference was found in smoking behaviour during a 5-year period between the CT group versus the control group.

\section{Why read on?}

- Overall, we found an increasing smoking cessation rate during this screening programme, higher motivation to quit at baseline predicted smoking abstinence at the final screening round.

therefore be lost. On the other hand, screening may provide an opportunity to counsel participants about smoking cessation. ${ }^{9}{ }^{10}$ There is, therefore, a need for randomised controlled trials in which the smoking habits of screened participants are compared to a non-screened group, so that the actual effect of screening on smoking behaviour can be evaluated. Only few randomised trials have investigated this by analysing smoking habits in a selected cohort of participants in the CT and control groups. ${ }^{10}$ To the best of our knowledge, this study is the first large-scale investigation of smoking behaviour of participants undergoing a complete 5 -year screening programme.

We set up the Danish Lung Cancer Screening Trial (DLCST), a 5-year screening trial, and have previously reported the results of smoking habits during the first 2 years of screening (at baseline and year 2$)^{11}$; during this period, there was no sign of either increased or reduced smoking in screened participants compared with a control group, that is, no significant difference in smoking habits between the screening and control groups. Our results were comparable with findings reported by other groups. ${ }^{10}$ The DLCST found a net quit rate of $6 \%$ after 1 year of screening, but it was unclear whether this was a short-term smoking cessation effect or whether this effect would also persist throughout the future screening rounds.

The purpose of this paper is to present and analyse smoking habits during all five screening rounds in the DLCST. 


\section{METHODS}

The study population comprised participants from the DLCST, a 5-year randomised trial that examined the effect of annual screening with low-dose CT on lung cancer mortality. ${ }^{12}$ Participants were randomised to either annual screening by low-dose CT of the chest (CT group) or no intervention (control group), by a computer programme (random permuted blocks of 10 participants with double blinded block size). Individuals volunteered for the DLCST by responding to advertisements in free local and regional newspapers. The study enrolled current or ex-smokers aged 50-70 years, with a smoking history of more than 20 pack-years. Ex-smokers had to have quit smoking after the age of 50 years, and less than 10 years ago. The study was conducted at Gentofte University Hospital, Copenhagen. A more detailed description of the DLCST study design, and inclusion and exclusion criteria, has been published elsewhere. ${ }^{12} 13$ The CT group underwent five annual CT scans of the thorax during the study, while the control group had neither CT scans nor chest radiographs. Both groups attended the same number of annual visits, at which smoking status was determined along with other assessments.

Between 1 November 2004 and 31 March 2006, a total of 4104 participants (mean age 58 years; $45 \%$ women) were enrolled. Smoking habits (self-reported) were assessed annually, starting with an initial (baseline) screening and followed by four annual screening rounds, giving a total of five screening years. At baseline and the second screening visit, self-reported smoking habits were objectively verified by measuring carbon monoxide (CO) levels in exhaled breath. ${ }^{11} 1415$

Current smokers were defined as participants who were actively smoking within the 4 weeks prior to the annual visit, and ex-smokers were defined as subjects with self-declared smoking abstinence for at least 4 weeks prior to the annual visit and with an exhaled CO $<10 \mathrm{ppm}$. Any self-reported ex-smokers with a CO level above $10 \mathrm{ppm}$ were excluded from analysis as they were considered as smokers.

At baseline, motivation to quit smoking among current smokers was assessed using the question 'How strong is your motivation to quit smoking?', with responses rated from 1 (no motivation) to 5 (very high motivation). ${ }^{16} 17$ At the final (year 5) screening, the frequency of smokers and ex-smokers was related to the distribution of baseline smoker motivation to quit using Model 1 (see 'Statistics' below).

\section{Counselling}

At each annual visit, participants in the CT group and the control group were given minimal smoking cessation counselling (less than $5 \mathrm{~min}$ ) by nurses who were certified in smoking cessation and had at least 3 years experience of counselling. Smokers were informed about the dangers of smoking and received brief advice to quit together with a brochure about smoking cessation. Ex-smokers were encouraged to remain abstinent.

\section{STATISTICS}

Annual smoking status and the missing recordings of smoking status were compared between the CT group and the control group using $\chi^{2}$ test. Motivation to quit at baseline was compared with smoking status at year 5 of screening using $\chi^{2}$ and logistic regression analysis by using this model: smoking status at year 5 $=$ motivation to quit smoking at baseline. Analyses were performed using R-statistical software V.2.14.0, and a $\mathrm{p}$ value of 0.05 or less was considered statistically significant. Analyses were performed using R-statistical software V.2.14.0, and a $\mathrm{p}$ value of 0.05 or less was considered statistically significant.

Smoking status at the final screening round (year 5) was calculated using two different models:

1. Missing values not included in the analysis (Model 1).

2. Missing data on smoking status at year 5 classified as the last known smoking status from previous screening rounds (Model 2).

\section{RESULTS}

Baseline characteristics are shown in table 1.The progression of the 4104 subjects randomised to either annual CT $(n=2052)$ or no intervention $(n=2052)$ through the 5 -year study is shown in figure 1.

\section{Annual smoking status}

Table 2 shows the annual smoking status from baseline up to the fifth year of screening for the CT group and control group. Five ex-smokers had an exhaled $\mathrm{CO}$ value above $10 \mathrm{ppm}$ during the first year of screening and were excluded from the analyses. There was no significant difference in smoking habits between the two groups during the study.

Of the 3124 smokers at baseline (in the CT + control groups), 1583 (51\%) remained smokers from baseline until the final screening round at year 5. A total of 980 subjects were ex-smokers at baseline, of whom 672 (69\%) remained ex-smokers throughout all five screening rounds. Overall, $45 \%$ (1849/4104) of participants changed their smoking habits during the study period.

Annual point prevalence quit rate among baseline smokers increased from $11 \%$ after 2 years to $24 \%$ after 5 years. For the ex-smokers at baseline, the annual point prevalence relapse rate to smoking was $9 \%$ after 2 years and $10 \%$ after 5 years (table 3).

\section{Missing recordings of smoking status}

Missing recording of smoking status was mainly due to loss to follow-up increased from $8.6 \%$ at baseline to $14.6 \%$ at the final screening round (year 5), and were more frequent in the control group $(12 \%)$ than the CT group $\left(6 \% ; \mathrm{p}<0.001, \chi^{2}\right.$ test $)$. To analyse the effect of missing values on smoking status at the final (year 5) screening, the two previously described models were used, and the results are presented in table 4 .

No significant difference between the CT group and control group was observed either when the missing values were not included (Model 1) or when all missing values were set to the last known smoking status (Model 2). Since we found no difference between the CT group and control group in Model 1 and Model 2, the overall (CT + control) rate of ex-smokers was determined: 24\% (baseline), 28\% (year 2), 33\% (year 3), 35\%

Table 1 Baseline characteristics of the participants in the Danish Lung Cancer Screening Trial (DLCST)

\begin{tabular}{lll}
\hline Parameter & CT group & Control group \\
\hline Gender (M/F) & $1147 / 905$ & $1120 / 932$ \\
Mean age (years) (range) & $57.9(49-71)$ & $57.8(49-71)$ \\
Mean pack years (SD) & $36.4(13.4)$ & $35.9(13.4)$ \\
Mean age (years) at smoking start (SD) & $17.0(3.8)$ & $17.1(5.7)$ \\
Mean cigarettes per day (SD) & $19.1(4.4)$ & $18.8(4.3)$ \\
Smoking status smokers /ex-smokers & $1545 / 507$ & $1579 / 473$ \\
\hline
\end{tabular}




\section{CONSORT 2010 Flow Diagram}

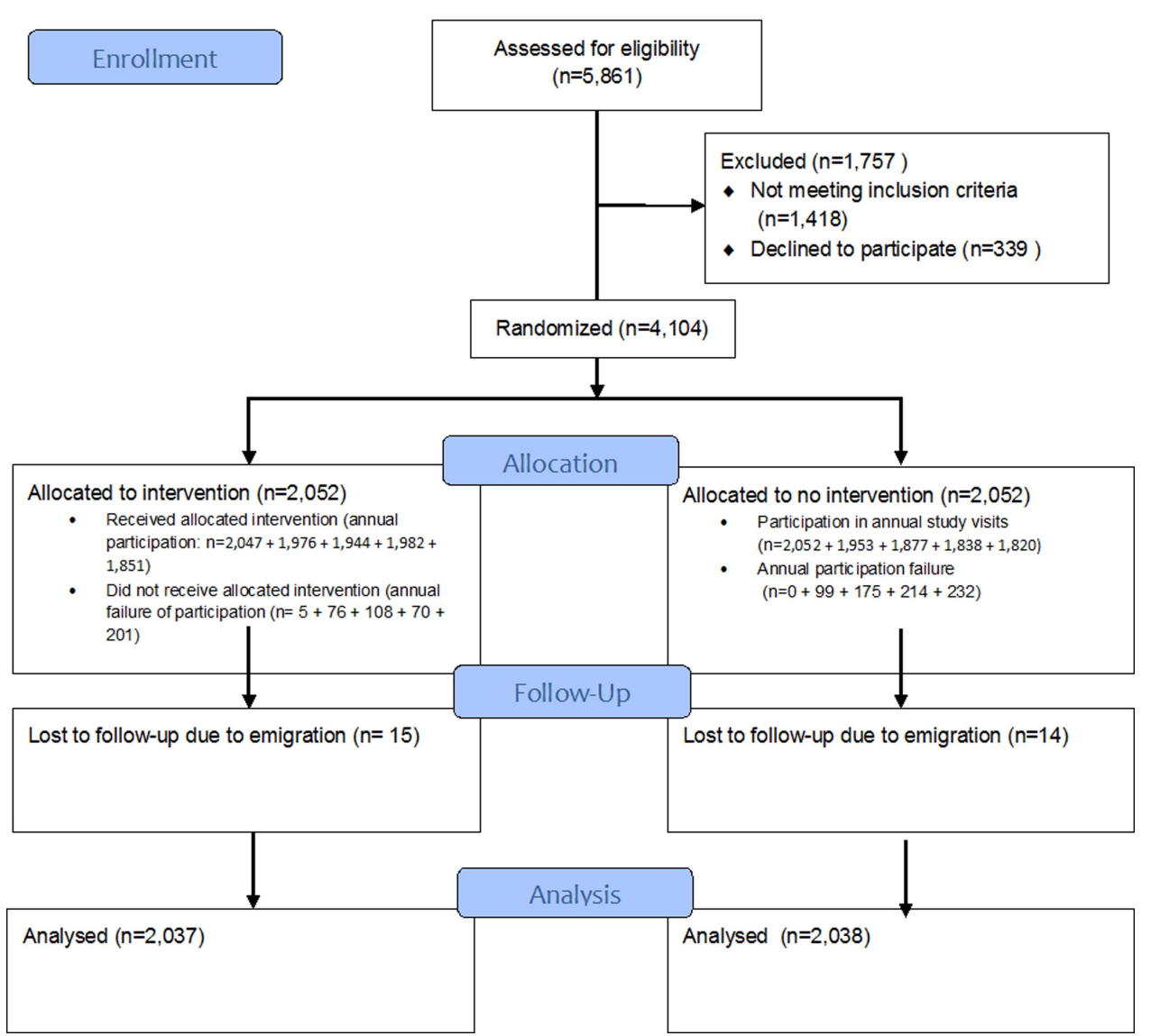

Figure 1 Disposition of participants in the CT group and control group throughout the 5-year study.

(year 4), and 37\% (year 5), $\left(\chi^{2} \mathrm{p}<0.001\right.$ ) (figure 2). In these analyses all missing values were regarded as current smokers (Model 2).

\section{Motivation to quit}

Table 5 shows the distribution of baseline motivation to quit in smokers and smoking status at the final screening (year 5). In these analyses, $17 \%$ of subjects had missing values and were not included in the calculations (Model 1).

Significantly more subjects who were ex-smokers at the year 5 screening were either highly $(37 \%)$ or very highly $(17 \%)$ motivated to quit smoking at baseline compared with subjects who smoked at year 5 screening (33\% and $12 \%$, respectively).
Correspondingly, significantly fewer subjects who were ex-smokers at the year 5 screening had rated their motivation to quit at baseline as either absent (9\%), very low (9\%) or low (28\%) compared with subjects who were smoking at the year 5 screening (12\%, 10\% and 33\%, respectively) In the logistic regression analysis, very high $(\mathrm{OR}=1.98)$ and high $(\mathrm{OR}=1.55)$ motivation to quit smoking at baseline was significantly correlated with smoking cessation at year 5 of screening (table 5).

\section{DISCUSSION}

Overall, the proportion of ex-smokers (CT + control group) significantly increased from $24 \%$ at baseline to $37 \%$ at the year 5 screening $(p<0.001)$, but we found no statistically significant

Table 2 Annual smoking status from baseline up to the year 5 screening for the CT group and control group

\begin{tabular}{|c|c|c|c|c|c|c|}
\hline & \multicolumn{2}{|l|}{$\mathrm{CT}$ group } & \multicolumn{2}{|l|}{ Control group } & \multirow[b]{2}{*}{ p Value* } & \multirow[b]{2}{*}{ Missing (\%) } \\
\hline & Smokers/ex-smokers & Ex-smokers (\%) & Smokers/ex-smokers & Ex-smokers (\%) & & \\
\hline Baseline & $1545 / 507$ & 25 & $1579 / 473$ & 23 & 0.213 & 0 \\
\hline Year 2 & $1335 / 596$ & 31 & $1274 / 540$ & 30 & 0.532 & 8.7 \\
\hline Year 3 & $1251 / 693$ & 36 & $1131 / 652$ & 37 & 0.559 & 9.2 \\
\hline Year 4 & $1132 / 756$ & 40 & $1038 / 683$ & 40 & 0.827 & 12.1 \\
\hline Year 5 & $1051 / 806$ & 43 & $937 / 713$ & 43 & 0.909 & 14.6 \\
\hline
\end{tabular}


Table 3 Annual point prevalence of quitters and relapsers to smoking, expressed as absolute numbers and as percentage of baseline smokers $(n=3124)$ and baseline ex-smokers $(n=980)$

\begin{tabular}{|c|c|c|c|c|c|c|}
\hline & $\begin{array}{l}\text { Point } \\
\text { prevalence } \\
\text { quitters (N) }\end{array}$ & $\begin{array}{l}\text { Per } \\
\text { cent }\end{array}$ & $\begin{array}{l}p \\
\text { Value* }\end{array}$ & $\begin{array}{l}\text { Point } \\
\text { prevalence } \\
\text { ex-smokers } \\
\text { who relapsed } \\
\text { (N) }\end{array}$ & $\begin{array}{l}\text { Per } \\
\text { cent }\end{array}$ & $\begin{array}{l}p \\
\text { Value* }\end{array}$ \\
\hline Year 2 & 339 & 11 & $<0.001$ & 91 & 9 & 0.287 \\
\hline Year 3 & 551 & 18 & & 115 & 12 & \\
\hline Year 4 & 661 & 21 & & 109 & 11 & \\
\hline Year 5 & 743 & 24 & & 98 & 10 & \\
\hline
\end{tabular}

differences in annual smoking status between the CT group and control group.

Screening with low-dose CT had no direct effect on the smoking status of the participants, and did not promote more smoking, as one might fear when conducting a lung cancer screening programme. No significant difference in smoking behaviour was observed between the CT group and the control group throughout all five annual screening rounds in the DLCST. We had previously published smoking habits from the first 2 years of the DLCST, which also showed no significant difference between the CT and control groups. ${ }^{11}$ This does not seem to be a short-term effect as no statistically significant differences in smoking habits emerged between the CT and control groups throughout all five annual screening rounds. In published smoking cessation studies-often with 1-year follow-upmissing values are typically in the range of $35-50 \%,{ }^{18-21}$ which seems higher than that observed in our 5-year study; therefore, missing values in our study seem to be acceptably low. We analysed smoking habits by excluding (Model 1) and including (Model 2) missing values; both models confirmed that there was no difference in the change of smoking habits between the CT and control groups.

The Nelson Study is another large randomised lung cancer screening trial which has investigated smoking habits of screening participants. They reported a 2 -year point prevalence quit rate of $14.5 \%$ in the intervention arm versus $19.1 \%$ in the control arm, but after including missing values there was no statistical difference among the two arms. ${ }^{10}$ The Nelson Study control group differed as they had no clinic visits and was only followed by phone calls, so the reliability regarding smoking status might be lower than in the intervention group. The absolute quit rate was in accordance with the findings in our study here.

Smoking habits were validated using exhaled CO during the first two screening rounds, and only a negligible number of subjects who appeared to have lied about their smoking status were

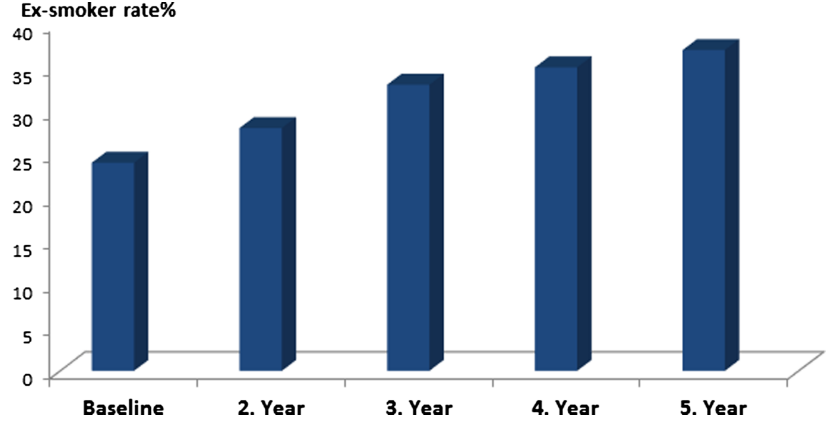

Figure 2 The overall (CT group plus control group) rate of ex-smokers in the Danish Lung Cancer Screening Trial (DLCST). Missing values were not included in the analysis (Model 1).

detected. We believe this was due to the fact that the DLCST was not primarily a smoking cessation trial and, therefore, participants were more honest with regards to their smoking habits. During screening rounds where smoking habits were not validated using exhaled $\mathrm{CO}$, there was no dramatic increase in quit rates (figure 2), suggesting that the 'liar rate' was still negligible even when $\mathrm{CO}$ validation was not used to verify self-reported smoking status.

The study has its limitation. Study participants volunteered to be part of a screening trial and may, therefore, be subject to selection bias since participants who volunteer for clinical trials may be more motivated to stop smoking than the general population. This selection bias is also known to be related to social class, although there was no difference in social class between our CT and control groups (data not shown). ${ }^{22}$ Our study may also be biased, as current smokers may tend to miss follow-up visits more frequently than non-smokers because they do not wish to be confronted with their smoking. However, as smoking cessation was not the primary focus of the DLCST (the focus was on early lung cancer detection), we do not suspect that the changes in smoking habits are different among participants who attended follow-up and those lost to follow-up. Our analyses of missing values support this hypothesis (table 4).

Previously when we published results of analyses of smoking habits after 1-year screening, ${ }^{11}$ we found that a positive finding on the CT (which resulted in a 3-month rescan), significantly promoted a higher quit rate and a lower relapse rate. However, this effect could not be found when we analysed all five screening rounds by comparing smoking habits of those with and without CT findings (data not shown). This could suggest, that the previously reported effect may be a transient short-term effect of a positive finding on CT, but without having a longlasting significant effect during the 5 -year screening programme.

Overall, approximately $45 \%$ of participants in the DLCST changed their smoking behaviour during the 5 -year study, and we observed a net quit rate of $17 \%$. The ex-smoker rate

Table 4 Analyses of smoking habits at the final (year 5) screening round using two different calculation methods: Model 1: missing values not included in analysis and Model 2: missing smoking status at year 5 classified as last known smoking status from previous screening rounds

\begin{tabular}{llllcccc}
\hline & CT group & Ex-smokers (\%) & Missing values (\%) & Control group & Ex-smokers (\%) & Missing values (\%) & $p$ Value* \\
\hline Baseline: Smokers/ex-smokers & $1545 / 507$ & 25 & 0 & $1579 / 473$ & 23 & 0 & 0.213 \\
Model 1: Smokers/ex-smoker & $1051 / 806$ & 43 & 10 & $937 / 713$ & 43 & 20 & 0.909 \\
Model 2: Smokers/ex-smoker & $1188 / 864$ & 42 & 0 & $1244 / 808$ & 40 & 0 & 0.075 \\
\hline${ }^{*} \chi^{2}$ Test. & & & & &
\end{tabular}


Table 5 Motivation to quit among smokers at baseline compared with smoking status for these individuals at the final year 5 screening round

\begin{tabular}{|c|c|c|c|c|c|c|c|}
\hline \multirow[b]{2}{*}{$\begin{array}{l}\text { Motivation at } \\
\text { baseline }\end{array}$} & \multicolumn{2}{|c|}{$\begin{array}{l}\text { Smokers at } \\
\text { year } 5\end{array}$} & \multicolumn{2}{|c|}{$\begin{array}{l}\text { Ex-smokers } \\
\text { at year } 5\end{array}$} & \multirow[b]{2}{*}{$\begin{array}{l}p \\
\text { Value* }\end{array}$} & \multirow[b]{2}{*}{ OR } & \multirow[b]{2}{*}{$\begin{array}{l}p \\
\text { Valuet }\end{array}$} \\
\hline & $\mathrm{N}$ & $\begin{array}{l}\text { Per } \\
\text { cent }\end{array}$ & $N$ & $\begin{array}{l}\text { Per } \\
\text { cent }\end{array}$ & & & \\
\hline Very high & 218 & 11.7 & 125 & 17.1 & $<0.001$ & 1.98 & $<0.001$ \\
\hline High & 604 & 32.5 & 271 & 37.0 & & 1.55 & 0.005 \\
\hline Low & 619 & 33.3 & 208 & 28.4 & & 1.16 & 0.336 \\
\hline Very low & 190 & 10.2 & 63 & 8.6 & & 1.15 & 0.501 \\
\hline None & 228 & 12.3 & 66 & 9.0 & & 1 & \\
\hline
\end{tabular}

OR, OR for smoking cessation.

Per cent show column percentages of each motivation level in both smoking status groups.

${ }^{*} \chi^{2}$ Test.

†Logistic regression model: smoking status at 5 year=motivation at baseline. 'None'

motivation set as reference group.

increased significantly across the CT group and control group, from $24 \%$ at baseline to $37 \%$ at the final screening round 5 years later. Participation in the DLCST seemed to be a successful method of promoting smoking cessation compared to the spontaneous quit rate in the general Danish population. We calculated a reasonable age, sex and regional matched control population from a Danish survey of smoking habits over the same 5 -year period, which showed a decrease in the prevalence of daily smokers from approximately $29 \%$ in 2005 to $26 \%$ in $2010 .^{23}$ This $3 \%$ decrease in smoking prevalence in the general Danish population-matched control population, compared with the $13 \%$ decrease in the DLCST, justifies our conclusion that participation in the DLCST, per se, probably resulted in the relatively high quit rate. As previously mentioned, the DLCST focused on early detection of lung cancer; it was not primarily a smoking cessation study, and only minimal smoking cessation counselling was offered. Nonetheless, we found a point prevalence quit rate of $24 \%$ (smokers at baseline who were ex-smokers at year 5), while the relapse rate of ex-smokers at baseline remained stable at around 11\% (table 3). This outcome is in accordance with the quit rate reported in many prospective smoking cessation studies that employ more cessation support combined with smoking cessation medications. ${ }^{24}$

Greater emphasis on smoking cessation in the DLCST, with more cessation support and smoking cessation medications, may have resulted in an even higher cessation rate. Interestingly, approximately $50 \%$ of participants in our study had no or very low motivation to quit smoking at baseline. Higher motivation to quit at baseline was significantly linked to smoking abstinence at the final screening round (table 5). Overall, our results show that baseline motivation was a predictor of long-term abstinence. As approximately half the smokers in the DLCST were highly or very highly motivated to quit at baseline, it would probably be feasible to enrol a large proportion of these smokers in a formal smoking cessation programme. In this context, lung cancer screening with a CT scan could be a window of opportunity for promoting smoking cessation. Therefore, we propose that smoking cessation should be an integral part of screening trials for lung cancer.

\section{CONCLUSION}

Our results did not indicate that CT scanning, per se, affected the smoking behaviour of participants in a lung cancer screening trial. However, participation in this screening trial alone significantly promoted smoking cessation in the CT group and the control group as the ex-smoker rate increased from $28 \%$ to $37 \%$ during the 5 -year study period. At the fifth year of screening, we found a $24 \%$ point prevalence quit rate among participants who were smoking at baseline; a quit rate of this magnitude is much higher than expected in the general population. Higher motivation to quit at baseline predicted smoking abstinence at the final screening round.

Acknowledgements The authors wish to thank the DLCST steering committee, including Birgit Guldhammer Skov MD, Karen Bach MD, Jann Mortensen MD, Hanne Thorsen MD, John Brodersen MD, Niels Seersholm MD, Hanne Hansen MD, Klaus Fuglsang Kofoed MD, Paul Clementsen MD and Klaus Richter Larsen MD.

Contributors HA and PT have drafted the manuscript. All authors revised the manuscript. Guarantor: HA and PT.

Funding The Danish Lung Cancer Screening Trial (DLCST) is funded in full by a governmental grant by the Danish Ministry of Health and Prevention.

\section{Competing interests None.}

Ethics approval Ethics approval was provided by Committee of Copenhagen County and the Danish Data Protection Agency.

Provenance and peer review Not commissioned; externally peer reviewed.

\section{REFERENCES}

1 Xu DM, Gietema $H$, de Koning $H$, et al. Nodule management protocol of the NELSON randomised lung cancer screening trial. Lung Cancer 2006;54:177-84.

2 Infante M, Cavuto S, Lutman FR, et al. A randomized study of lung cancer screening with spiral computed tomography. Three-year results from the DANTE Trial. Am J Respir Crit Care Med 2009;180:445-53.

3 Becker N, Delorme S, Kauczor HU. LUSI: the German component of the European trial on the efficacy of multislice-CT for the early detection of lung cancer. Onkologie 2008;31:P0320.

4 Henschke $\mathrm{Cl}$, Yankelevitz DF, Libby DM, et al. The international early lung cancer action program investigators. Survival of patients with Stage I lung cancer detected on CT screening. N Engl J Med 2006;355:1763-71.

5 Swensen SJ, Jett JR, Hartman TE, et al. CT Screening for lung cancer: five-year prospective experience. Radiology 2005;235:259-65.

6 Aberle DR, Adams AM, Berg CD, et al. Reduced lung-cancer mortality with low-dose computed tomographic screening. N Engl J Med 2011;365:395-409.

7 http://www.reuters.com/article/2010/12/17/us-lung-canceridUSTRE6BG64720101217

8 Taylor KL, Sanderson C, Zinckea N, et al. Lung cancer screening as a teachable moment for smoking cessation. Lung Cancer 2007;56:125-34.

9 Bach PB, Mirkin JN, Oliver TK, et al. Benefits and harms of CT screening for lung cancer. A systematic review. JAMA 2012;307:2418-29.

10 van der Aalst CM, van den Bergh KAM, Willemsen MC, et al. Lung cancer screening and smoking abstinence: 2 year follow-up data from the Dutch-Belgian randomised controlled lung cancer screening trial. Thorax 2010;65:600-5.

11 Ashraf $\mathrm{H}$, Tønnesen P, Pedersen $\mathrm{JH}$, et al. Smoking habits were unaffected by CT screening at 1-year follow-up in the Danish Lung Cancer Screening Trial (DLCST). Thorax 2009;64:371-2.

12 Pedersen JH, Ashraf H, Dirksen A, et al. The Danish Randomized Lung Cancer CT Screening Trial-Overall design and results of the prevalence round. J Thorac Oncol 2009;4:608-14

13 Saghir Z, Ashraf H, Dirksen A, et al. Contamination during 4 years of annual CT screening in the Danish Lung Cancer Screening Trial (DLCST). Lung Cancer 2011;71:323-7.

14 Kentala J, Utriainen P, Pahkala K, et al. Verification of adolescent self-reported smoking. Addict Behav 2004;29:405-11.

15 Morabia A, Bernstein MS, Curtin F, et al. Validation of self-reported smoking status by simultaneous measurement of carbon monoxide and salivary thiocyanate. Prev Med 2001;32:82-8.

16 Tønnesen P, Mikkelsen K, Markholst C, et al. Nurse-conducted smoking cessation with minimal intervention in a lung clinic: a randomized controlled study. Eur Respir J 1996;9:2351-5.

17 Thyrian JR, Panagiotakos DB, Polychronopoulos E, et al. The relationship between smokers' motivation to quit and intensity of tobacco control at the population level: a comparison of five European countries. http://www.biomedcentral.com/1471-2458/8/2

18 Gonzales D, Rennard SI, Nides M, et al. Varenicline, an alfa4beta2 nicotinic acetylcholine receptor partial agonist, vs. sustained- release bupropion and placebo for smoking cessation. JAMA 2006;296:47-55.

19 Jorenby DE, Hays T, Rigotti NA, et al. Efficacy of varenicline, an alfa4, beta2 nicotinic acetylcholine receptor partial agonist, vs. placebo or sustained-release bupropion for smoking cessation. JAMA 2006;296:56-63. 
20 Tønnesen P, Lauri $\mathrm{H}$, Perfekt $\mathrm{R}$, et al. Efficacy of a nicotine mouth spray in smoking cessation: a randomised, double-blind trial. Eur Respir $\int$ 2012:40:548-54.

21 Tønnesen P, Paoletti P, Gustavsson G, et al. Higher dosage nicotine patches increase one-year smoking cessation rates: results from the European CEASE trial. Eur Respir J 1999;13:238-46.
22 Hestbech MS, Siersma V, Dirksen A, et al. Participation bias in a randomised trial of screening for lung cancer. Lung Cancer 2011;73:325-31.

23 The Danish National Health Board Annual Smoking Prevalence Survey. http://www.sst.dk

24 Fiore MC, Bailey WC, Cohen SJ, et al. Treating tobacco use and dependence. Clinical practice guideline. Rockville, MD: U.S. Department of Health and Human Services. Public Health Service, 2008. http://www.ahrq/gov/path/tobacco.htm clinic 